InIFolPharm

Therapiemanagement bei Eisenüberladung

\title{
Niedriger Serumferritin-Wert begünstigt die Prognose
}

\author{
Regelmäßige Transfusionen mit Erythrozytenkonzentraten (EK) führen \\ bei Patienten mit myelodysplastischem Syndrom (MDS) zu einer \\ Eisenüberladung des Organismus. Die medikamentöse Eisenchelation \\ ist daher ein wichtiger Therapiebaustein für MDS-Patienten. Eine \\ Option: Deferasirox.
}

Besonders profitieren MDS-Patienten von der Eisenchelation, bei denen es zu einem deutlichen Abfall des Serumferritin-Werts kommt. Der Abfall des Serumferritins korreliert mit dem hämatologischen Ansprechen, erläuterte Norbert Gattermann, Düsseldorf.

Der positive Einfluss der Eisenchelation wurde beispielsweise in der prospektiv randomisierten Phase-III-Studie EPIC (Evaluation of Patients Iron Chelation) gezeigt. Hier verbesserte die Eisenchelation mit Deferasirox (Exjade ${ }^{\circledast}$ ) die Hämatopoese der Patienten und reduzierte den Transfusionsbedarf innerhalb weniger Monate deutlich [Gattermann $\mathrm{N}$ et al.
Leukemia Res. 2010;34(9):1143-50]. Besonders groß war dieser Einfluss auf die Hämatopoese bei jenen Patienten, die infolge einer effektiven Deferasirox-Behandlung eine deutliche Serumferritin-Reduktion aufwiesen [Gattermann $\mathrm{N}$ et al. Haematologica. 2012;97(9):1364-71]. Eine prospektive Analyse untermauert diese Daten [de Swart L et al. ASH Annual Meeting 2011; Abstract 2775]: Danach haben Patienten mit einem SerumferritinWert unter $300 \mathrm{ng} / \mathrm{ml}$ eine besonders günstige Prognose. Aber auch Patienten mit einem Serumferritin-Wert unter 1.000 $\mathrm{ng} / \mathrm{ml}$ leben signifikant länger als jene mit einem Wert über $1.000 \mathrm{ng} / \mathrm{ml}(\mathrm{p}<0,0001)$.
Die Effektivität der Eisenchelation hängt von der verabreichten Dosis und der Compliance des Patienten ab, so Christian Junghanß, Rostock. Die empfohlene Anfangsdosis für Deferasirox beträgt $20 \mathrm{mg} / \mathrm{kg}$ Körpergewicht (KG). Je nach Transfusionsrate und Therapieziel müsse die Dosis jedoch angepasst werden, z. B. auf $30 \mathrm{mg} / \mathrm{kg} \mathrm{KG}$ bei Patienten mit mehr als vier EK-Einheiten pro Monat. Mathias Schmid, Zürich, Schweiz, empfiehlt, den Eisenchelator abends 30 Minuten vor oder drei Stunden nach einer Mahlzeit in Wasser, Saft oder Tee aufzulösen und einzunehmen.

Birgit-Kristin Pohlmann

Satellitensymposium „Kontroverse klinische Kasuistiken - Therapiemanagement bei Eisenüberladung" im Rahmen der DGHO-Jahrestagung 2012, Stuttgart, 19.10.2012; Veranstalter: Novartis Pharma

\section{Neue Therapiechance für Patienten mit ALK-positivem nichtkleinzelligen Lungenkarzinom}

\section{Für Patienten mit ALK-positivem nichtkleinzelligen Lungen- karzinom (NSCLC) ermöglicht Crizotinib eine personalisierte Therapie. Vor der Behandlung ist der Nachweis des ALK- Rearrangements erforderlich.}

Bei etwa 3-5\% aller NSCLC-Patienten lassen sich ALK-Genrearrangements nachweisen [Sasaki T et al. Eur J Cancer. 2010;46(10):1773-80]. In der Regel handelt es sich um EML4-ALK-Translokationen. Die Folge: „EML4-ALK-Fusionsproteine sind konstitutiv aktiv“, erklärte Frank Griesinger, Oldenburg. „Somit kommt es zu einer permanenten Proliferation und zu einer Hemmung der Apoptose." Crizotinib (Xalkori ${ }^{\circledR}$ ) ist ein oraler, kompetitiver Inhibitor der ALK-Tyrosinkinase. Dadurch werden die permanente Proliferation der Tumorzellen und die Unterdrückung der Apoptose gehemmt.

Die bedingte Zulassung von Crizotinib basiert auf den Ergebnissen zweier einarmiger Studien bei vorbehandelten
Patienten mit fortgeschrittenem ALKpositiven NSCLC. In einer Phase-I-Studie erreichte Crizotinib (2x $250 \mathrm{mg} / \mathrm{d}$ ) eine objektive Ansprechrate (ORR) von $61 \%$, eine Krankheitskontrolle (DCR) über acht Wochen von $84 \%$ und ein progressionsfreies Überleben (PFS) von 9,2 Monaten [Camidge R et al. ASCO 2011; Abstract 2501]. Eine Phase-II-Studie ( $\mathrm{n}=$ 261) führte $z u$ ähnlichen Ergebnissen (ORR $53 \%$, PFS 8,5 Monate, DCR $85 \%$ ) [Kim DW et al. ASCO 2012; Abstract 7533]. Median dauerte das Ansprechen in den beiden Studien 48 bzw. 46 Wochen.

Die Interimsergebnisse einer PhaseIII-Studie belegen die Überlegenheit von Crizotinib gegenüber einer Standard-
Second-Line-Chemotherapie mit Pemetrexed oder Docetaxel bei Patienten mit ALK-positivem NSCLC in Stadium IIIB/ IV. Das mediane PFS betrug 7,7 Monate im Crizotinib-Arm gegenüber 3 Monaten im Chemotherapie-Arm (Hazard Ratio [HR] 0,49; p < 0,0001). Auch die Ansprechrate unter Crizotinib war höher (ORR 65,3 vs. 19,5\%).

Wichtig sei die frühzeitige molekulare Testung von Patienten mit NSCLC auf eine ALK-Mutation, so Manfred Dietel, Berlin. Da jeder Patient ein ALK-Rearrangement im Tumor haben könne, sollten die Patienten nicht aufgrund ihres Alters, Geschlechts oder Raucherstatus selektiert werden.

Abdol Ameri

Pressekonferenz "Zielgerichtet effektiv - Crizotinib (Xalkori ${ }^{\oplus}$ ) beim vorbehandelten ALK-positiven fortgeschrittenen nicht-kleinzelligen Lungenkarzinom (NSCLC)“, Berlin, 11.12.2012; Veranstalter: Pfizer Pharma 